\title{
ENTREPRENEURIAL CAPABILITY AND ENTREPRENEURIAL SUCCESS
}

\author{
Oyeku Oyedele M. \\ Department of Extension \& Linkage, Federal Institute of Industrial Research Oshodi, \\ P.M.B 21023, Ikeja, Lagos, Nigeria
}

\section{Oduyoye Oluseyi}

Business administration \& Marketing Department, Babcock Business School, Babcock University, Ilishan Remo, Ogun State, Nigeria.

\section{Karimu Funmilayo Ajoke}

Techno-entrepreneurship \& Techno-economics Division, Federal Institute of Industrial Research Oshodi, P.M.B 21023, Ikeja, Lagos, Nigeria

\section{Akindoju F. Akinfolarin}

Techno-entrepreneurship \& Techno-economics Division, Federal Institute of Industrial Research Oshodi, P.M.B 21023, Ikeja, Lagos, Nigeria

\section{Togunde Mumeen Oyekola}

Techno-entrepreneurship \& Techno-economics Division, Federal Institute of Industrial Research Oshodi, P.M.B 21023, Ikeja, Lagos, Nigeria

\section{Elemo G. N.}

Federal Institute of Industrial Research Oshodi, P.M.B 21023, Ikeja, Lagos, Nigeria

\begin{abstract}
The purpose of this study is to examine the effect of entrepreneurial capability on entrepreneurial success. Nine thousand, four hundred and fifty (9,450) small and medium enterprises (SMEs) who are registered members of National Association of Small and Medium Enterprises (NASME), National Association of Small Scale Industrialists (NASSI) and Association of Small Business Owners in Nigeria (ASBON) in Lagos State is the sample population for the study. Proportionate stratified random sampling technique is used to select sample size of 381 determined using the formula developed by the National Education Association (1960) for sample size determination. Research instrument is questionnaire. Primary data on the dependent variable (Entrepreneurial success) and independent variable (Entrepreneurial capability) are collected using questionnaire this research instrument. Entrepreneurial capability measures are entrepreneurial orientation and entrepreneurial self efficacy while measures for entrepreneurial success are profitability, market share, net asset growth, sales growth and government policies reflecting both financial and non financial measures. Fifty selected SMEs are used in a pilot study to pretest the research instrument. Analysis of data from the pilot study gives Cronbach's Alpha values of 0.664, 0.795 and 0.85 for entrepreneurial orientation, entrepreneurial self efficacy and entrepreneurial success
\end{abstract}


respectively. The result of the regression analysis indicated that entrepreneurial capability has a strong positive relationship with entrepreneurial success $(R=.475)$. The coefficient of determination $R^{2}$ is .226, suggests that $22.6 \%$ of the variation (or changes) in entrepreneurial success of selected SMEs in Lagos State is explained by entrepreneurial capability. The value of $F(1,208)=60.605, p<.05$, demonstrates that entrepreneurial capability statistically and significantly predicts entrepreneurial success. This is a clear indication that entrepreneurial capability significantly influences entrepreneurial success in Lagos State. The coefficient of entrepreneurial capability is statistically significant $(t=7.785, p<.05)$. The regression model explaining the results is given by: ENT_SU $=-5.065+0.624 E N T$ CAP. Based on the findings, the null hypothesis $\left(\mathrm{H}_{04}\right)$ which states that entrepreneurial capability has no significant effect on entrepreneurial success is rejected.

KEYWORDS: Entrepreneurial success, entrepreneurial capability, entrepreneurial self-efficacy.

\section{INTRODUCTION}

Researchers in the field of entrepreneurship study are yet to reach a widely acceptable agreement on the concept of entrepreneurial capability and it is doubtful if an acceptable concept could be reached. This is because researchers view things from different perspectives. While some researchers view entrepreneurial capability from the angle of characteristics and traits of individual entrepreneurs (Oyeku, Oduyoye, Elemo, Akindoju and Karimu, 2014; Ohyama, Braguinsky and Murphy, 2004; Xu Yan-Mei, 2013; Staniewski, Janowski, and Awruk, 2016; Ayala and Manzano, 2014; Windapo, 2018; Razak, Said, Ahmad, and Jumain, 2017; Scheers, 2016) that could be deployed to enhance enterprise success others look at it beyond the context of individual entrepreneurs but with application to entrepreneurs working as a team or corporate entrepreneurs (Phillips and Tracey, 2007). The recent trend however, in entrepreneurial process is to go beyond the description of entrepreneurial capability in terms of entrepreneurial traits and entrepreneurial personality but to specify specific abilities and resources needed to commence any entrepreneurial activity.

Some researchers on entrepreneurial capability have attempted studies on the whole process view of entrepreneurs' characteristics in new ventures (Obschonka, M., et al, 2011) while others concentrate on different aspects like entrepreneurial opportunity (Zhang, Tansuhaj and McCulloughe, 2009), learning (Jiao, Ogilvie and Cui, 2010), contextual factors (Zhang, et al., 2010), and network relationships (Clarysse, Tartari and Salter, 2011).

Several researchers have examined factors that influence success of enterprise including entrepreneurial capability (Oyeku et al., 2014; Oyeku, Oduyoye, Ashikia, Kabuoh and Elemo, 2014; Woldesenbet, Ran and Jones, 2012; Man, Lau and Chan, 2002; Man, Lau and Chan, 2000; Zhang, Tansuhaj and McCulloughe, 2009; Zahra, Abdelgawad and Tsang, 2011; and 
Abdelgawad, et al., 2013). In doing these, researchers have adopted different measures to study entrepreneurial capability. He Xiao-Gang and Lin Gu-Yan (2009) studied entrepreneurial capability using the measures of strategic capability, management capability, relationship ability, innovation capability, the capability to seize opportunities, and learning capability with empirical evidence that entrepreneurial capability promotes corporate performance. Yang Yang (2014) studied the relationship between entrepreneurial capability and corporate performance; the results showed a significant positive correlation between entrepreneurial capability and corporate performance.

Yao Xiang and Xu Yan-Mei (2013) considered that entrepreneurs are at the heart of a network formed by an enterprise's own characteristics and development needs; as opined that entrepreneurs can use various key knowledge and resources in the network to meet the needs of their enterprise based on the influencing mechanism of entrepreneurial capability on enterprise innovation and corporate performance.

For the purpose of this study, entrepreneurial capability is defined as the internal ability of the entrepreneur that is required to start and operate a successful enterprise expressed in terms of entrepreneurial orientation and entrepreneurial self-efficacy. The objective of this study is to examine the effect of entrepreneurial capability on entrepreneurial success of small and medium enterprises in Lagos. The measures for entrepreneurial capability are entrepreneurial orientation and entrepreneurial self efficacy.

\section{METHODOLOGY}

\section{Sample, Procedures and Measures}

The population for this study is small and medium enterprises (SMEs) who are registered members of the National Association of Small and Medium Enterprises (NASME), National Association of Small Scale Industrialists (NASSI) and Association of Small Business Owners of Nigeria (ASBON) in Lagos State. There 9,450 registered members all together in these three Business Membership Organizations (BMOs) which presents the study population. These enterprises engage in nines types of enterprises namely: food \& beverages, construction, consultancy, education, computer services, manufacturing, retailing, healthcare, and others.

The method for sample selection from the sampling frame is proportionate stratified random sampling technique. The first stage in sample selection is stratifying the sample population into the following nine strata based on the types of the enterprises: Education, Food and Beverage, Manufacturing/production (non-food and beverage), Services (including consultancy, media), Computer (Internet/IT) and electronics, Construction (including construction materials), Health care, Retail/Sales and Others. The second stage is sample size determination. Sample size of 381 was determined using the formula for sample size determination developed by the National Education Association (1960). The samples are then selected proportionately from all the strata 
based on the sample size using simple random sampling technique. Research instrument for primary data collection is questionnaire. Primary data on the dependent variable (Entrepreneurial success) and independent variable (entrepreneurial capability) are collected with the aid of the research instrument.

Measures for entrepreneurial capability are entrepreneurial orientation and entrepreneurial self efficacy. The New General Self Efficacy Scale (NGSE) questionnaire on entrepreneurial self efficacy (Chen, Gully and Eden, 2001) was adapted with additional six items to the 8-item scale NGSE. The first six items on the new general scale are classified as optimism-related items while the last two are classified as overconfidence- related items. Additional six items are included as overconfidence-related items to obtain a modified 14-item general scale for entrepreneurial self efficacy (MNGSE). The 8-item NGSE scale has been used mostly by researchers to measure entrepreneurial intention rather that entrepreneurial success.

Entrepreneurial Orientation questions are adapted from Miller and Friesen (1982) and Covin and Slevin (1989). There are basically eight items in this instrument but the study added two items to make ten questions solicit responses from respondents on the three measures of entrepreneurial orientation namely: innovativeness, proactiveness and risk taking. Five entrepreneurial success measures namely: profitability, sales growth, government policies, market share and net asset growth used in this study included both financial and non-financial measures reported in literature (Wiklund, 1999; Butler, Keh \& Chamommam, 2000; Murphy \& Callaway, 2004). In all, there are 16-entrepreneurial success related questions in the research instrument.

The research instrument is pretested to reduce measurement errors and improve the validity of the construct (Dillman, 2000) using a pilot study with 50 samples. Analysis of data from the pilot study shows that Cronbach's Alpha value for entrepreneurial orientation, entrepreneurial self efficacy and entrepreneurial success are $0.664,0.795$ and 0.85 respectively which are considered good (Islam et al, 2011).

\section{Statistical Procedures}

Different statistical packages, structural and mathematical model, as well as specially developed software have been used by researchers in the field of entrepreneurial studies for data analysis. (Sorensen, 2007; Dyer, Greggersen \& Christensen, 2008; Perez \& Canino, 2009; Sarwoko et al, 2013; Kotey et al, 2013; Torres \& Watson, 2013; Owoseni \& Akanbi, 2011; Setyawati et al, 2011; Wei-Wen Wu, 2009; Burger, O’Neill \& Mahadea, 2005; Buttner, 2001). This study employs Simple and Multiple Regression Analysis to predict the impact of the independent variable(s) on the dependent variable using Statistical Packages for Social Sciences (SPSS). 


\section{MODEL SPECIFICATIONS}

The relating equations are:

$\mathrm{Y}=\mathrm{f}(\mathrm{X})$, where: $\mathrm{Y}=$ Entrepreneurial Success (ENT_SU) and $\mathrm{X}$ is Entrepreneurial Capability (ENT_CAP). ENT_CAP is measured by Entrepreneurial Orientation (ENT_ORIEN) and Entrepreneurial Self Efficacy (ENT_SELF).

From the hypotheses:

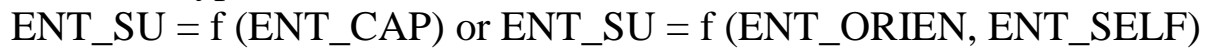

The implicit form of the functional relationship of the variables expressed above is:

ENT_SU $=\alpha 0+\beta 1 E N T \_O R I E N+\beta 2 E N T \_S E L F+$ e where $\beta 1-\beta 2$ are coefficients of independent variables and $\mathrm{e}$ is the error term.

\section{RESULTS}

Research Objective: To examine the effect of entrepreneurial capability on entrepreneurial success.

Research Question: What is the effect of entrepreneurial capability on entrepreneurial success?

Table 1: Descriptive statistics of opinions of respondents on entrepreneurial orientation

\begin{tabular}{|c|c|c|c|c|c|c|c|c|c|}
\hline $\begin{array}{l}\text { Entrepreneurial } \\
\text { Orientation }\end{array}$ & UD & SD & DA & PD & PA & $\mathbf{A}$ & SA & $\bar{X}$ & SD \\
\hline $\begin{array}{l}\text { In my company, } \\
\text { there exist a very } \\
\text { strong emphasis on } \\
\text { R\&D, } \\
\text { technological } \\
\text { leadership and } \\
\text { innovations. }\end{array}$ & $\begin{array}{c}16 \\
7.6 \%\end{array}$ & $\begin{array}{c}2 \\
1.0 \%\end{array}$ & $\begin{array}{c}16 \\
7.6 \\
\%\end{array}$ & $\begin{array}{c}14 \\
6.7 \%\end{array}$ & $\begin{array}{c}30 \\
14.3 \%\end{array}$ & $\begin{array}{c}48 \\
22.9 \\
\%\end{array}$ & $\begin{array}{c}84 \\
40.0 \\
\%\end{array}$ & $\begin{array}{r}4 . \\
48\end{array}$ & $\begin{array}{r}1.8 \\
15\end{array}$ \\
\hline $\begin{array}{l}\text { My company } \\
\text { introduced many } \\
\text { new lines of } \\
\text { products or services } \\
\text { in the past five } \\
\text { years. }\end{array}$ & $\begin{array}{c}20 \\
9.5 \%\end{array}$ & $\begin{array}{c}0 \\
0.0 \%\end{array}$ & $\begin{array}{c}22 \\
10.5 \\
\%\end{array}$ & $\begin{array}{c}22 \\
10.5 \%\end{array}$ & $\begin{array}{c}32 \\
15.2 \%\end{array}$ & $\begin{array}{c}50 \\
23.8 \\
\%\end{array}$ & $\begin{array}{c}64 \\
30.5 \\
\%\end{array}$ & $\begin{array}{r}4 . \\
15\end{array}$ & $\begin{array}{r}1.8 \\
70\end{array}$ \\
\hline $\begin{array}{l}\text { The changes in } \\
\text { product lines } \\
\text { (types/number of } \\
\text { products) for my } \\
\text { company have } \\
\text { usually been } \\
\text { dramatic. }\end{array}$ & $\begin{array}{c}16 \\
7.6 \%\end{array}$ & $\begin{array}{c}10 \\
4.8 \%\end{array}$ & $\begin{array}{c}16 \\
7.6 \\
\%\end{array}$ & $\begin{array}{c}26 \\
12.4 \%\end{array}$ & $\begin{array}{c}54 \\
25.7 \%\end{array}$ & $\begin{array}{c}54 \\
25.7 \\
\%\end{array}$ & $\begin{array}{c}34 \\
16.2 \\
\%\end{array}$ & $\begin{array}{r}3 . \\
86\end{array}$ & $\begin{array}{r}1.7 \\
25\end{array}$ \\
\hline
\end{tabular}


European Journal of Business and Innovation Research

Vol.8, No.5, pp. 56-79, August 2020

Published by ECRTD-UK

Print ISSN: 2053-4019(Print), Online ISSN: 2053-4027(Online)

\begin{tabular}{|c|c|c|c|c|c|c|c|c|c|}
\hline $\begin{array}{l}\text { I reward employees } \\
\text { who find creative } \\
\text { ways of improving } \\
\text { company's } \\
\text { performance. }\end{array}$ & $\begin{array}{c}8 \\
3.8 \%\end{array}$ & $\begin{array}{c}8 \\
3.8 \%\end{array}$ & $\begin{array}{c}8 \\
3.8 \\
\%\end{array}$ & $\begin{array}{c}12 \\
5.7 \%\end{array}$ & $\begin{array}{c}50 \\
23.8 \%\end{array}$ & $\begin{array}{c}66 \\
31.4 \\
\%\end{array}$ & $\begin{array}{c}58 \\
27.6 \\
\%\end{array}$ & $\begin{array}{r}4 . \\
47\end{array}$ & $\begin{array}{r}1.5 \\
41\end{array}$ \\
\hline $\begin{array}{l}\text { I decide to adopt } \\
\text { new ideas only on } \\
\text { the basis of their } \\
\text { relative cost and } \\
\text { benefits to the } \\
\text { organization. }\end{array}$ & $\begin{array}{c}4 \\
1.9 \%\end{array}$ & $\begin{array}{c}2 \\
1.0 \%\end{array}$ & $\begin{array}{c}2 \\
1.0 \\
\%\end{array}$ & $\begin{array}{c}18 \\
8.6 \%\end{array}$ & $\begin{array}{c}38 \\
18.1 \%\end{array}$ & $\begin{array}{c}56 \\
26.7 \\
\%\end{array}$ & $\begin{array}{c}90 \\
42.9 \\
\%\end{array}$ & $\begin{array}{r}4 . \\
91\end{array}$ & $\begin{array}{r}1.2 \\
91\end{array}$ \\
\hline $\begin{array}{l}\text { My company is } \\
\text { typically the first to } \\
\text { initiate actions to } \\
\text { competitors, for } \\
\text { which competitors } \\
\text { then respond. }\end{array}$ & $\begin{array}{c}22 \\
10.5 \\
\%\end{array}$ & $\begin{array}{c}24 \\
11.4 \%\end{array}$ & $\begin{array}{c}22 \\
10.5 \\
\%\end{array}$ & $\begin{array}{c}22 \\
10.5 \%\end{array}$ & $\begin{array}{c}42 \\
20.0 \%\end{array}$ & $\begin{array}{c}52 \\
24.8 \\
\%\end{array}$ & $\begin{array}{c}26 \\
12.4 \\
\%\end{array}$ & $\begin{array}{r}3 . \\
42\end{array}$ & $\begin{array}{r}1.9 \\
11\end{array}$ \\
\hline $\begin{array}{l}\text { Very often, my } \\
\text { company is the first } \\
\text { company to } \\
\text { introduce new } \\
\text { products/services, } \\
\text { techniques, } \\
\text { technologies etc. }\end{array}$ & $\begin{array}{c}22 \\
10.5 \\
\%\end{array}$ & $\begin{array}{c}34 \\
16.2 \%\end{array}$ & $\begin{array}{c}20 \\
9.5 \\
\%\end{array}$ & $\begin{array}{c}34 \\
16.2 \%\end{array}$ & $\begin{array}{c}28 \\
13.3 \%\end{array}$ & $\begin{array}{c}34 \\
16.2 \\
\%\end{array}$ & $\begin{array}{c}38 \\
18.1 \\
\%\end{array}$ & $\begin{array}{r}3 . \\
27\end{array}$ & $\begin{array}{r}2.0 \\
11\end{array}$ \\
\hline $\begin{array}{l}\text { While my project } \\
\text { idea may not } \\
\text { entirely be new, I } \\
\text { am thinking of new } \\
\text { and better ways to } \\
\text { make it } \\
\text { competitive. }\end{array}$ & $\begin{array}{c}10 \\
4.8 \%\end{array}$ & $\begin{array}{c}4 \\
1.9 \%\end{array}$ & $\begin{array}{c}2 \\
1.0 \\
\%\end{array}$ & $\begin{array}{c}8 \\
3.8 \%\end{array}$ & $\begin{array}{c}24 \\
11.4 \%\end{array}$ & $\begin{array}{c}80 \\
38.1 \\
\%\end{array}$ & $\begin{array}{c}82 \\
39.0 \\
\%\end{array}$ & $\begin{array}{r}4 . \\
86\end{array}$ & $\begin{array}{r}1.4 \\
93\end{array}$ \\
\hline $\begin{array}{l}\text { I have strong } \\
\text { preference for high } \\
\text { risk projects with } \\
\text { chances of very } \\
\text { high return. }\end{array}$ & $\begin{array}{c}8 \\
3.8 \%\end{array}$ & $\begin{array}{c}8 \\
3.8 \%\end{array}$ & $\begin{array}{c}10 \\
4.8 \\
\%\end{array}$ & $\begin{array}{c}8 \\
3.8 \%\end{array}$ & $\begin{array}{c}50 \\
23.8 \%\end{array}$ & $\begin{array}{c}72 \\
34.3 \\
\%\end{array}$ & $\begin{array}{c}54 \\
25.7 \\
\%\end{array}$ & $\begin{array}{r}4 . \\
46\end{array}$ & $\begin{array}{r}1.5 \\
34\end{array}$ \\
\hline
\end{tabular}


Vol.8, No.5, pp. 56-79, August 2020

Published by ECRTD-UK

Print ISSN: 2053-4019(Print), Online ISSN: 2053-4027(Online)

\begin{tabular}{|c|c|c|c|c|c|c|c|c|c|}
\hline $\begin{array}{l}\text { When confronted } \\
\text { with decision } \\
\text { making situations } \\
\text { involving } \\
\text { uncertainty, my } \\
\text { firm typically adopt } \\
\text { a cautious, "wait } \\
\text { and see" posture in } \\
\text { order to minimize } \\
\text { the probability of } \\
\text { making costly } \\
\text { decisions. }\end{array}$ & $\begin{array}{c}0 \\
0.0 \%\end{array}$ & $\begin{array}{c}72 \\
34.3 \%\end{array}$ & $\begin{array}{c}44 \\
21.0 \\
\%\end{array}$ & $\begin{array}{c}12 \\
5.7 \%\end{array}$ & $\begin{array}{c}10 \\
4.8 \%\end{array}$ & $\begin{array}{c}6 \\
2.9 \\
\%\end{array}$ & $\begin{array}{c}66 \\
31.4 \\
\%\end{array}$ & $\begin{array}{r}3 . \\
15\end{array}$ & $\begin{array}{r}2.1 \\
38\end{array}$ \\
\hline $\begin{array}{l}\text { I believe that, } \\
\text { owing to the nature } \\
\text { of environment, } \\
\text { bold, wide ranging } \\
\text { acts are necessary } \\
\text { to achieve the } \\
\text { firm's objectives. }\end{array}$ & $\begin{array}{c}10 \\
4.8 \%\end{array}$ & $\begin{array}{c}0 \\
0.0 \%\end{array}$ & $\begin{array}{c}10 \\
4.8 \\
\%\end{array}$ & $\begin{array}{c}4 \\
1.9 \%\end{array}$ & $\begin{array}{c}28 \\
13.3 \%\end{array}$ & $\begin{array}{c}78 \\
37.1 \\
\%\end{array}$ & $\begin{array}{c}80 \\
38.1 \\
\%\end{array}$ & $\begin{array}{r}4 . \\
83\end{array}$ & $\begin{array}{r}1.4 \\
80\end{array}$ \\
\hline
\end{tabular}

Key: UD (Undecided), SD (Strongly Disagree), D (Disagree), PD (Partially Disagree), PA (Partially Agree), A (Agree) and SA (Strongly Agree).

Table 1 question (1) on innovativeness indicated that 16 respondents representing $7.6 \%$ where unsure that there exist a very strong emphasis on research and development, technological leadership and innovations in their company, 2 respondents representing $1.0 \%$ strongly disagreed, 16 respondents representing $7.6 \%$ disagreed, 14 respondents representing $6.7 \%$ partially disagreed, 30 respondents representing $14.3 \%$ partially agreed, 48 respondents representing $22.9 \%$ agreed and 84 respondents representing $40.0 \%$ strongly agreed.

Question (2) on innovativeness showed that 20 respondents representing 9.5\% were uncertain that their company introduced many new lines of products or services in the past five years, 22 respondents representing $10.5 \%$ disagreed, 22 respondents representing $10.5 \%$ partially disagreed, 32 respondents representing $15.2 \%$ partially agreed, 50 respondents representing 23.8\% Agreed and 64 respondents representing 30.5\% strongly agreed.

Question (3) on innovativeness indicated that 16 respondents representing $7.6 \%$ were unsure that changes in product lines for their company have usually been dramatic, 10 respondents representing $4.8 \%$ strongly disagreed, 16 respondents representing $7.6 \%$ disagreed, 26 respondents representing $12.4 \%$ partially disagreed, 54 respondents representing $25.7 \%$ partially agreed, 54 respondents representing $25.7 \%$ agreed and 34 respondents representing 16.2\% strongly agreed. 
Question (4) on innovativeness showed that 8 respondents representing 3.8\% were unsure that their company rewards creativeness of employees, 8 respondents representing $3.8 \%$ strongly disagreed, 8 respondents representing $3.8 \%$ disagreed, 12 respondents representing $5.7 \%$ partially disagreed, 50 respondents representing $23.8 \%$ partially agreed, 66 respondents representing $31.4 \%$ agreed and 58 respondents representing $27.6 \%$ strongly agreed.

Question (5) on innovativeness indicated that 4 respondents representing 1.9\% were uncertain in adopting new ideas only on the basis of their relative cost and benefits to the organization, 2 respondents representing $1.0 \%$ strongly disagreed, 2 respondents representing $1.0 \%$ disagreed, 18 respondents representing $8.6 \%$ partially disagreed, 38 respondents representing $18.1 \%$ partially agreed, 56 respondents representing 26.7\% Agreed and 90 respondents representing $42.9 \%$ strongly agreed.

Question (6) on pro-activeness indicated that 22 respondents representing $10.5 \%$ were unsure that their company is typically the first to initiate actions to competitors, for which competitors then respond, 24 respondents representing $11.4 \%$ strongly disagreed, 22 respondents representing $10.5 \%$ disagreed, 22 respondents representing $10.5 \%$ partially disagreed, 42 respondents representing $20.0 \%$ partially agreed, 52 respondents representing $24.8 \%$ agreed and 26 respondents representing $12.4 \%$ strongly agreed.

Question (7) on pro-activeness showed that 22 respondents representing $10.5 \%$ were uncertain that very often their company is the first company to introduce new products/services, techniques, technologies etc. 34 respondents representing $16.2 \%$ strongly disagreed, 20 respondents representing $9.5 \%$ disagreed, 34 respondents representing $16.2 \%$ partially disagreed, 28 respondents representing $13.3 \%$ partially agreed, 34 respondents representing $16.2 \%$ agreed and 38 respondents representing $18.1 \%$ strongly agreed.

Question (8) on pro-activeness indicated that 10 respondents representing $4.8 \%$ were unsure that while their project idea may not entirely be new, that they are thinking of new and better ways to make it competitive, 4 respondents representing $1.9 \%$ strongly disagreed, 2 respondents representing $1.0 \%$ disagreed, 8 respondents representing $3.8 \%$ partially disagreed, 24 respondents representing $11.4 \%$ partially agreed, 80 respondents representing $38 \%$ agreed and 82 respondents representing $39.0 \%$ strongly agreed.

Question (9) on risk taking indicated that 8 respondents representing 3.8\% were unsure that they have strong preference for high risk projects with chances of very high return, 8 respondents representing $3.8 \%$ strongly disagreed, 10 respondents representing $4.8 \%$ disagreed, 8 respondents representing $3.8 \%$ partially disagreed, 50 respondents representing $23.8 \%$ partially agreed, 72 respondents representing $34.3 \%$ agreed and 54 respondents representing $25.7 \%$ strongly agreed. 
Question (10) on risk taking showed that 72 respondents representing 34.3\% strongly disagreed that when they are confronted with decision making situations involving uncertainty, their firm typically adopt a cautious, "wait and see" posture in order to minimize the probability of making costly decisions, 44 respondents representing $21.0 \%$ disagreed, 12 respondents representing $5.7 \%$ partially disagreed, 10 respondents representing $4.8 \%$ partially agreed, 6 respondents representing $2.9 \%$ agreed and 66 respondents representing $31.4 \%$ strongly agreed.

Question (11) on risk taking indicated that 10 respondents representing $4.8 \%$ were unsure that they believe that, owing to the nature of environment, bold, wide ranging acts are necessary to achieve the firm's objectives, 10 respondents representing 4.8\% disagreed, 4 respondents representing $1.9 \%$ partially disagreed, 28 respondents representing $13.3 \%$ partially agreed, 78 respondents representing $37.1 \%$ agreed and 80 respondents representing $38.1 \%$ strongly agreed.

Table 2: Descriptive statistics of opinions of respondents on entrepreneurial self-efficacy

\begin{tabular}{|c|c|c|c|c|c|c|c|c|c|}
\hline $\begin{array}{l}\text { Entrepreneurial } \\
\text { Self Efficacy }\end{array}$ & UD & SD & DA & PD & PA & A & SA & $X$ & SD \\
\hline $\begin{array}{l}\text { I will be able to } \\
\text { achieve most goals } \\
\text { I have set for } \\
\text { myself. }\end{array}$ & $\begin{array}{c}2 \\
1.0 \%\end{array}$ & $\begin{array}{c}0 \\
0.0 \%\end{array}$ & $\begin{array}{c}0 \\
0.0 \%\end{array}$ & $\begin{array}{c}2 \\
1.0 \%\end{array}$ & $\begin{array}{c}40 \\
19.0 \%\end{array}$ & $\begin{array}{l}84 \\
40 . \\
0 \%\end{array}$ & $\begin{array}{c}82 \\
39.0 \\
\%\end{array}$ & 5.13 & .918 \\
\hline $\begin{array}{l}\text { When facing } \\
\text { difficult tasks, I } \\
\text { am certain that I } \\
\text { will accomplish } \\
\text { them. }\end{array}$ & $\begin{array}{c}0 \\
0.0 \%\end{array}$ & $\begin{array}{c}0 \\
0.0 \%\end{array}$ & $\begin{array}{c}0 \\
0.0 \%\end{array}$ & $\begin{array}{c}4 \\
1.9 \%\end{array}$ & $\begin{array}{c}40 \\
19.0 \%\end{array}$ & $\begin{array}{l}88 \\
41 . \\
9 \%\end{array}$ & $\begin{array}{c}78 \\
37.1 \\
\%\end{array}$ & 5.14 & .788 \\
\hline $\begin{array}{l}\text { In general, I think } \\
\text { that I can obtain } \\
\text { outcomes that are } \\
\text { important to me. }\end{array}$ & $\begin{array}{c}2 \\
1.0 \%\end{array}$ & $\begin{array}{c}4 \\
1.9 \%\end{array}$ & $\begin{array}{c}2 \\
1.0 \%\end{array}$ & $\begin{array}{c}2 \\
1.0 \%\end{array}$ & $\begin{array}{c}40 \\
19.0 \%\end{array}$ & $\begin{array}{l}100 \\
47 . \\
6 \%\end{array}$ & $\begin{array}{c}60 \\
28.6 \\
\%\end{array}$ & 4.92 & 1.069 \\
\hline $\begin{array}{l}\text { I don't think of } \\
\text { negative } \\
\text { consequences to } \\
\text { acts and make } \\
\text { decisions. }\end{array}$ & $\begin{array}{c}0 \\
0.0 \%\end{array}$ & $\begin{array}{c}24 \\
11.4 \%\end{array}$ & $\begin{array}{c}18 \\
8.6 \%\end{array}$ & $\begin{array}{c}34 \\
16.2 \%\end{array}$ & $\begin{array}{c}42 \\
20.0 \%\end{array}$ & $\begin{array}{l}50 \\
23 . \\
8 \%\end{array}$ & $\begin{array}{c}42 \\
20.0 \\
\%\end{array}$ & 3.96 & 1.607 \\
\hline $\begin{array}{l}\text { I don't express } \\
\text { skepticism about } \\
\text { possible impact of } \\
\text { new ideas to my } \\
\text { business } \\
\text { performance. }\end{array}$ & $\begin{array}{c}4 \\
1.9 \%\end{array}$ & $\begin{array}{c}26 \\
12.4 \%\end{array}$ & $\begin{array}{c}10 \\
4.8 \%\end{array}$ & $\begin{array}{c}16 \\
7.6 \%\end{array}$ & $\begin{array}{c}58 \\
27.6 \%\end{array}$ & $\begin{array}{l}60 \\
28 . \\
6 \%\end{array}$ & $\begin{array}{c}36 \\
17.1 \\
\%\end{array}$ & 4.01 & 1.634 \\
\hline
\end{tabular}


European Journal of Business and Innovation Research

Vol.8, No.5, pp. 56-79, August 2020

Published by ECRTD-UK

Print ISSN: 2053-4019(Print), Online ISSN: 2053-4027(Online)

\begin{tabular}{|c|c|c|c|c|c|c|c|c|c|}
\hline $\begin{array}{l}\text { I don't allow } \\
\text { myself to think of } \\
\text { the future of my } \\
\text { business as dim } \\
\text { and gloomy. }\end{array}$ & $\begin{array}{c}4 \\
1.9 \%\end{array}$ & $\begin{array}{c}10 \\
4.8 \%\end{array}$ & $\begin{array}{c}12 \\
5.7 \%\end{array}$ & $\begin{array}{c}12 \\
5.7 \%\end{array}$ & $\begin{array}{c}28 \\
13.3 \%\end{array}$ & $\begin{array}{l}68 \\
32 . \\
4 \%\end{array}$ & $\begin{array}{c}76 \\
36.2 \\
\%\end{array}$ & 4.66 & 1.539 \\
\hline $\begin{array}{l}\text { I believe I can } \\
\text { succeed at most } \\
\text { any endeavor to } \\
\text { which I set my } \\
\text { mind. }\end{array}$ & $\begin{array}{c}0 \\
0.0 \%\end{array}$ & $\begin{array}{c}4 \\
1.9 \%\end{array}$ & $\begin{array}{c}2 \\
1.0 \%\end{array}$ & $\begin{array}{c}10 \\
4.8 \%\end{array}$ & $\begin{array}{c}26 \\
12.4 \%\end{array}$ & $\begin{array}{l}78 \\
37 . \\
1 \%\end{array}$ & $\begin{array}{c}90 \\
42.9 \\
\%\end{array}$ & 5.10 & 1.062 \\
\hline $\begin{array}{l}\text { I will be able to } \\
\text { successfully } \\
\text { overcome many } \\
\text { challenges. }\end{array}$ & $\begin{array}{c}0 \\
0.0 \%\end{array}$ & $\begin{array}{c}0 \\
0.0 \%\end{array}$ & $\begin{array}{c}2 \\
1.0 \%\end{array}$ & $\begin{array}{c}4 \\
1.9 \%\end{array}$ & $\begin{array}{c}28 \\
13.3 \%\end{array}$ & $\begin{array}{l}106 \\
50 . \\
5 \%\end{array}$ & $\begin{array}{c}70 \\
33.3 \\
\%\end{array}$ & 5.13 & .783 \\
\hline $\begin{array}{l}\text { I am confident that } \\
\text { I can perform } \\
\text { effectively on } \\
\text { many different } \\
\text { tasks. }\end{array}$ & $\begin{array}{c}0 \\
0.0 \%\end{array}$ & $\begin{array}{c}0 \\
0.0 \%\end{array}$ & $\begin{array}{c}4 \\
1.9 \%\end{array}$ & $\begin{array}{c}10 \\
4.8 \%\end{array}$ & $\begin{array}{c}28 \\
13.3 \%\end{array}$ & $\begin{array}{l}98 \\
46 . \\
7 \%\end{array}$ & $\begin{array}{c}70 \\
33.3 \\
\%\end{array}$ & 5.05 & .911 \\
\hline $\begin{array}{l}\text { Compared to other } \\
\text { people, I can do } \\
\text { most tasks very } \\
\text { well. }\end{array}$ & $\begin{array}{c}2 \\
1.0 \%\end{array}$ & $\begin{array}{c}2 \\
1.0 \%\end{array}$ & $\begin{array}{c}4 \\
1.9 \%\end{array}$ & $\begin{array}{c}8 \\
3.8 \%\end{array}$ & $\begin{array}{c}38 \\
18.1 \%\end{array}$ & $\begin{array}{l}110 \\
52 . \\
4 \%\end{array}$ & $\begin{array}{c}46 \\
21.9 \\
\%\end{array}$ & 4.82 & 1.042 \\
\hline $\begin{array}{l}\text { Even when things } \\
\text { are tough, I can } \\
\text { perform quite } \\
\text { well. }\end{array}$ & $\begin{array}{c}0 \\
0.0 \%\end{array}$ & $\begin{array}{c}2 \\
1.0 \%\end{array}$ & $\begin{array}{c}4 \\
1.9 \%\end{array}$ & $\begin{array}{c}18 \\
8.6 \%\end{array}$ & $\begin{array}{c}30 \\
14.3 \%\end{array}$ & $\begin{array}{l}110 \\
52 . \\
4 \%\end{array}$ & $\begin{array}{c}46 \\
21.9 \\
\%\end{array}$ & 4.81 & .999 \\
\hline $\begin{array}{l}\text { I tend to } \\
\text { overestimate my } \\
\text { capacities for } \\
\text { succeeding in any } \\
\text { business. }\end{array}$ & $\begin{array}{c}0 \\
0.0 \%\end{array}$ & $\begin{array}{c}12 \\
5.7 \%\end{array}$ & $\begin{array}{c}14 \\
6.7 \%\end{array}$ & $\begin{array}{c}30 \\
14.3 \%\end{array}$ & $\begin{array}{c}48 \\
22.9 \%\end{array}$ & $\begin{array}{l}74 \\
35 . \\
2 \%\end{array}$ & $\begin{array}{c}32 \\
15.2 \\
\%\end{array}$ & 4.21 & 1.360 \\
\hline $\begin{array}{l}\text { I don't doubt my } \\
\text { ability to cope } \\
\text { under new, } \\
\text { untested } \\
\text { conditions. }\end{array}$ & $\begin{array}{c}0 \\
0.0 \%\end{array}$ & $\begin{array}{c}6 \\
2.9 \%\end{array}$ & $\begin{array}{c}14 \\
6.7 \%\end{array}$ & $\begin{array}{c}18 \\
8.6 \%\end{array}$ & $\begin{array}{c}48 \\
22.9 \%\end{array}$ & $\begin{array}{l}74 \\
35 . \\
2 \%\end{array}$ & $\begin{array}{c}50 \\
23.8 \\
\%\end{array}$ & 4.52 & 1.284 \\
\hline
\end{tabular}




\begin{tabular}{|l|c|c|c|c|c|c|c|c|c|}
\hline When I do & 0 & 0 & 2 & 12 & 26 & 84 & 86 & 5.14 & .912 \\
$\begin{array}{l}\text { something, I see to } \\
\text { it that it doesn't } \\
\text { only get done but } \\
\text { done excellently. }\end{array}$ & $0.0 \%$ & $0.0 \%$ & $1.0 \%$ & $5.7 \%$ & $12.4 \%$ & 40. & 41.0 & & \\
\hline
\end{tabular}

Key: UD (Undecided), SD (Strongly Disagree), D (Disagree), PD (Partially Disagree), PA (Partially Agree), A (Agree) and SA (Strongly Agree).

Table 2 question (1) on optimism indicates that 2 respondents representing $1.0 \%$ were unsure that they will be able to achieve most goals they have set for myself, 2 respondents representing $1.0 \%$ partially disagreed, 40 respondents representing $19.0 \%$ partially agreed, 84 respondents representing 40.0\% Agreed and 82 respondents representing 39.0\% strongly agreed.

Question (2) on optimism showed that 4 respondents representing $1.9 \%$ partially disagreed that when they are faced with difficult tasks, they are certain that they accomplished them, 40 respondents representing $19.0 \%$ partially agreed, 88 respondents representing $41.9 \%$ agreed and 78 respondents representing $37.1 \%$ strongly agreed.

Question (3) on optimism indicated that 2 respondents representing 1.0\% were uncertain that they can obtain outcomes that are important to them, 4 respondents representing $1.9 \%$ strongly disagreed, 2 respondents representing $1.0 \%$ disagreed, 2 respondents representing $1.0 \%$ partially disagreed, 40 respondents representing $19.0 \%$ partially agreed, 100 respondents representing $47.6 \%$ agreed and 60 respondents representing $28.6 \%$ strongly agreed.

Question (4) on optimism indicated that 24 respondents representing $11.4 \%$ strongly disagreed that they do not think of negative consequences while acts and make decisions, 18 respondents representing $8.6 \%$ disagreed, 34 respondents representing 16.2\% partially disagreed, 42 respondents representing $20.0 \%$ partially agreed, 50 respondents representing $23.8 \%$ agreed and 42 respondents representing $20.0 \%$ strongly agreed.

Question (5) on optimism showed that 4 respondents representing 1.9\% were unsure that they do not express skepticism about possible impact of new ideas to their business performance, 26 respondents representing $12.4 \%$ strongly disagreed, 10 respondents representing $4.8 \%$ disagreed, 16 respondents representing $6.7 \%$ partially disagreed, 58 respondents representing $7.6 \%$ partially agreed, 60 respondents representing $28.6 \%$ agreed and 36 respondents representing $17.1 \%$ strongly agreed.

Question (6) on optimism showed that 4 respondents representing 1.9\% were uncertain that they do not allow themselves to think of the future of their business as dim and gloomy, 10 respondents representing $4.8 \%$ strongly disagreed, 12 respondents representing $5.7 \%$ disagreed, 12 respondents representing $5.7 \%$ partially disagreed, 28 respondents representing $13.3 \%$ partially agreed, 68 respondents representing 32.4\% agreed and 76 respondents representing $36.2 \%$ strongly agreed. 
Question (7) on overconfidence indicated that 4 respondents representing $1.9 \%$ strongly disagreed that they believed they can succeed at most any endeavor to which they set their mind, 2 respondents representing $1.0 \%$ disagreed, 10 respondents representing $4.8 \%$ partially disagreed, 26 respondents representing $12.4 \%$ partially agreed, 78 respondents representing $37.1 \%$ agreed and 90 respondents representing $42.9 \%$ strongly agreed.

Question (8) on overconfidence showed that 2 respondents representing $1.0 \%$ disagreed that they would be able to successfully overcome many challenges, 4 respondents representing $1.9 \%$ partially disagreed, 28 respondents representing $13.3 \%$ partially agreed, 106 respondents representing $50.5 \%$ agreed and 70 respondents representing $33.3 \%$ strongly agreed.

Question (9) on overconfidence indicated that 4 respondents representing $1.9 \%$ disagreed that they are confident that they can perform effectively on many different tasks, 10 respondents representing $4.8 \%$ partially disagreed, 28 respondents representing $13.3 \%$ partially agreed, 98 respondents representing $46.7 \%$ agreed and 70 respondents representing $33.3 \%$ strongly agreed. Question (10) on overconfidence showed that 2 respondents representing $1.0 \%$ where unsure that when compared to other people, they can do most tasks very well, 2 respondents representing $1.0 \%$ strongly disagreed, 4 respondents representing $1.9 \%$ disagreed, 8 respondents representing $3.8 \%$ partially disagreed, 38 respondents representing $18.1 \%$ partially agreed, 110 respondents representing $52.4 \%$ agreed and 46 respondents representing $21.9 \%$ strongly agreed.

Question (11) on overconfidence indicated that 2 respondents representing $1.0 \%$ strongly disagreed that when things are tough, they performed quite well, 4 respondents representing $1.9 \%$ disagreed, 18 respondents representing $8.6 \%$ partially disagreed, 30 respondents representing $14.3 \%$ partially agreed, 110 respondents representing $52.4 \%$ agreed and 46 respondents representing $21.9 \%$ strongly agreed.

Question (12) on overconfidence indicated that 12 respondents representing $5.7 \%$ strongly disagreed that they tend to overestimate their capacities for succeeding in any business, 14 respondents representing $6.7 \%$ disagreed, 30 respondents representing $14.3 \%$ partially disagreed, 48 respondents representing $22.9 \%$ partially agreed, 74 respondents representing $35.2 \%$ agreed and 32 respondents representing $15.2 \%$ strongly agreed.

Question (13) on overconfidence indicated that 6 respondents representing $2.9 \%$ strongly disagreed that they do not doubt their ability to cope under new, untested conditions, 14 respondents representing $6.7 \%$ disagreed, 18 respondents representing $8.6 \%$ partially disagreed, 48 respondents representing $22.9 \%$ partially agreed, 74 respondents representing $35.2 \%$ agreed and 50 respondents representing $23.8 \%$ strongly agreed.

Question (14) on overconfidence showed that 2 respondents representing $1.0 \%$ disagreed that when they do something, they see to it that it does not only get done but done excellently, 12 
respondents representing $5.7 \%$ partially disagreed, 26 respondents representing $12.4 \%$ partially agreed, 48 respondents representing $40.0 \%$ agreed and 86 respondents representing $41.0 \%$ strongly agreed.

Table 3: Descriptive statistics of opinions of respondents on entrepreneurial success

\begin{tabular}{|c|c|c|c|c|c|c|c|c|c|}
\hline $\begin{array}{l}\text { Entrepreneurial } \\
\text { Success }\end{array}$ & UD & SD & DA & PD & PA & $\mathbf{A}$ & SA & $\bar{X}$ & SD \\
\hline $\begin{array}{l}\text { I am satisfied with the } \\
\text { profit level of my } \\
\text { business. }\end{array}$ & $\begin{array}{c}12 \\
5.7 \%\end{array}$ & $\begin{array}{c}34 \\
16.2 \%\end{array}$ & $\begin{array}{c}20 \\
9.5 \%\end{array}$ & $\begin{array}{c}30 \\
14.3 \%\end{array}$ & $\begin{array}{c}44 \\
21.0 \\
\% \\
\end{array}$ & $\begin{array}{c}48 \\
22.9 \\
\% \\
\end{array}$ & $\begin{array}{c}22 \\
10.5 \\
\% \\
\end{array}$ & 3.39 & 1.785 \\
\hline $\begin{array}{l}\text { The Company's' } \\
\text { profitability ratio such } \\
\text { as return on investment } \\
\text { and return on equity } \\
\text { showed that the firm is } \\
\text { making sustainable } \\
\text { profit. }\end{array}$ & $\begin{array}{c}12 \\
5.7 \%\end{array}$ & $\begin{array}{c}2 \\
1.0 \%\end{array}$ & $\begin{array}{c}20 \\
9.5 \%\end{array}$ & $\begin{array}{c}22 \\
10.5 \%\end{array}$ & $\begin{array}{c}46 \\
21.9 \\
\%\end{array}$ & $\begin{array}{c}76 \\
36.2 \\
\%\end{array}$ & $\begin{array}{c}32 \\
15.2 \\
\%\end{array}$ & 4.11 & 1.567 \\
\hline $\begin{array}{l}\text { Earnings per share } \\
\text { (EPS) of the firm have } \\
\text { increased. }\end{array}$ & $\begin{array}{c}20 \\
9.5 \%\end{array}$ & $\begin{array}{c}2 \\
1.0 \%\end{array}$ & $\begin{array}{c}24 \\
11.4 \%\end{array}$ & $\begin{array}{c}28 \\
13.3 \%\end{array}$ & $\begin{array}{c}46 \\
21.9 \\
\%\end{array}$ & $\begin{array}{c}60 \\
28.6 \\
\%\end{array}$ & $\begin{array}{c}30 \\
14.3 \\
\%\end{array}$ & 3.80 & 1.741 \\
\hline $\begin{array}{l}\text { The firms' overall } \\
\text { financial performance } \\
\text { has been } \\
\text { acknowledged by its } \\
\text { bank(s). }\end{array}$ & $\begin{array}{c}20 \\
9.5 \%\end{array}$ & $\begin{array}{c}6 \\
2.9 \%\end{array}$ & $\begin{array}{c}16 \\
7.6 \%\end{array}$ & $\begin{array}{c}36 \\
17.1 \%\end{array}$ & $\begin{array}{c}44 \\
21.0 \\
\%\end{array}$ & $\begin{array}{c}48 \\
22.9 \\
\%\end{array}$ & $\begin{array}{c}40 \\
19.0 \\
\%\end{array}$ & 3.82 & 1.797 \\
\hline $\begin{array}{l}\text { Since take off of my } \\
\text { business, sales level } \\
\text { has grown } \\
\text { significantly. }\end{array}$ & $\begin{array}{c}14 \\
6.7 \%\end{array}$ & $\begin{array}{c}0 \\
0.0 \%\end{array}$ & $\begin{array}{c}8 \\
3.8 \%\end{array}$ & $\begin{array}{c}22 \\
10.5 \%\end{array}$ & $\begin{array}{c}50 \\
23.8 \\
\%\end{array}$ & $\begin{array}{c}70 \\
33.3 \\
\%\end{array}$ & $\begin{array}{c}46 \\
21.9 \\
\%\end{array}$ & 4.32 & 1.562 \\
\hline $\begin{array}{l}\text { There has been an } \\
\text { increase in the number } \\
\text { of people who are } \\
\text { willing to sell and } \\
\text { patronize the } \\
\text { company's } \\
\text { goods/services. }\end{array}$ & $\begin{array}{c}14 \\
6.7 \%\end{array}$ & $\begin{array}{c}2 \\
1.0 \%\end{array}$ & $\begin{array}{c}6 \\
2.9 \%\end{array}$ & $\begin{array}{c}18 \\
8.6 \%\end{array}$ & $\begin{array}{c}32 \\
15.2 \\
\%\end{array}$ & $\begin{array}{c}92 \\
43.8 \\
\%\end{array}$ & $\begin{array}{c}46 \\
21.9 \\
\%\end{array}$ & 4.44 & 1.577 \\
\hline $\begin{array}{l}\text { The company's sales } \\
\text { strategy is responsible } \\
\text { for its increased } \\
\text { revenue through }\end{array}$ & $\begin{array}{c}16 \\
7.6 \%\end{array}$ & $\begin{array}{c}8 \\
3.8 \%\end{array}$ & $\begin{array}{c}4 \\
1.9 \%\end{array}$ & $\begin{array}{c}18 \\
8.6 \%\end{array}$ & $\begin{array}{c}40 \\
19.0 \\
\%\end{array}$ & $\begin{array}{c}86 \\
41.0 \\
\%\end{array}$ & $\begin{array}{c}38 \\
18.1 \\
\%\end{array}$ & 4.23 & 1.679 \\
\hline
\end{tabular}




\begin{tabular}{|c|c|c|c|c|c|c|c|c|c|}
\hline enhanced sales. & & & & & & & & & \\
\hline $\begin{array}{l}\text { Government policies } \\
\text { on patronage of made } \\
\text { in Nigeria goods is a } \\
\text { boost to sales in my } \\
\text { company. }\end{array}$ & $\begin{array}{c}22 \\
10.5 \\
\%\end{array}$ & $\begin{array}{c}6 \\
2.9 \%\end{array}$ & $\begin{array}{c}12 \\
5.7 \%\end{array}$ & $\begin{array}{c}16 \\
7.6 \%\end{array}$ & $\begin{array}{c}34 \\
16.2 \\
\%\end{array}$ & $\begin{array}{c}82 \\
39.0 \\
\%\end{array}$ & $\begin{array}{c}38 \\
18.1 \\
\%\end{array}$ & 4.06 & 1.834 \\
\hline $\begin{array}{l}\text { There has been a } \\
\text { significant increase in } \\
\text { the company's market } \\
\text { share. }\end{array}$ & $\begin{array}{c}18 \\
8.6 \%\end{array}$ & $\begin{array}{c}6 \\
2.9 \%\end{array}$ & $\begin{array}{c}8 \\
3.8 \%\end{array}$ & $\begin{array}{c}22 \\
10.5 \%\end{array}$ & $\begin{array}{c}60 \\
28.6 \\
\%\end{array}$ & $\begin{array}{c}60 \\
28.6 \\
\%\end{array}$ & $\begin{array}{c}36 \\
17.1 \\
\%\end{array}$ & 4.02 & 1.694 \\
\hline $\begin{array}{l}\text { The company has } \\
\text { expanded to other } \\
\text { products and markets. }\end{array}$ & $\begin{array}{c}14 \\
6.7 \%\end{array}$ & $\begin{array}{c}18 \\
8.6 \%\end{array}$ & $\begin{array}{c}22 \\
10.5 \%\end{array}$ & $\begin{array}{c}8 \\
3.8 \%\end{array}$ & $\begin{array}{c}60 \\
28.6 \\
\%\end{array}$ & $\begin{array}{c}58 \\
27.6 \\
\%\end{array}$ & $\begin{array}{c}30 \\
14.3 \\
\%\end{array}$ & 3.79 & 1.765 \\
\hline $\begin{array}{l}\text { The company's } \\
\text { product(s)/service(s) } \\
\text { have taken over a large } \\
\text { chunk of the market in } \\
\text { its immediate } \\
\text { environment and } \\
\text { beyond. }\end{array}$ & $\begin{array}{c}12 \\
5.7 \%\end{array}$ & $\begin{array}{c}8 \\
3.8 \%\end{array}$ & $\begin{array}{c}24 \\
11.4 \%\end{array}$ & $\begin{array}{c}28 \\
13.3 \%\end{array}$ & $\begin{array}{c}40 \\
19.0 \\
\%\end{array}$ & $\begin{array}{c}62 \\
29.5 \\
\%\end{array}$ & $\begin{array}{c}36 \\
17.1 \\
\%\end{array}$ & 3.93 & 1.685 \\
\hline $\begin{array}{l}\text { The company's } \\
\text { products/services now } \\
\text { enjoy a wider } \\
\text { acceptance compared } \\
\text { to when the } \\
\text { products/services were } \\
\text { introduced. }\end{array}$ & $\begin{array}{c}14 \\
6.7 \%\end{array}$ & $\begin{array}{c}4 \\
1.9 \%\end{array}$ & $\begin{array}{c}10 \\
4.8 \%\end{array}$ & $\begin{array}{c}18 \\
8.6 \%\end{array}$ & $\begin{array}{c}46 \\
21.9 \\
\%\end{array}$ & $\begin{array}{c}72 \\
34.3 \\
\%\end{array}$ & $\begin{array}{c}46 \\
21.9 \\
\%\end{array}$ & 4.28 & 1.634 \\
\hline $\begin{array}{l}\text { My business has } \\
\text { experienced } \\
\text { considerable growth in } \\
\text { net asset. }\end{array}$ & $\begin{array}{c}14 \\
6.7 \%\end{array}$ & $\begin{array}{c}2 \\
1.0 \%\end{array}$ & $\begin{array}{c}4 \\
1.9 \%\end{array}$ & $\begin{array}{c}20 \\
9.5 \%\end{array}$ & $\begin{array}{c}60 \\
28.6 \\
\%\end{array}$ & $\begin{array}{c}66 \\
31.4 \\
\%\end{array}$ & $\begin{array}{c}44 \\
21.0 \\
\%\end{array}$ & 4.30 & 1.547 \\
\hline $\begin{array}{l}\text { The net asset of the } \\
\text { company and its } \\
\text { liability are healthy } \\
\text { enough to guarantee } \\
\text { success. }\end{array}$ & $\begin{array}{c}14 \\
6.7 \%\end{array}$ & $\begin{array}{c}0 \\
0.0 \%\end{array}$ & $\begin{array}{c}14 \\
6.7 \%\end{array}$ & $\begin{array}{c}18 \\
8.6 \%\end{array}$ & $\begin{array}{c}56 \\
26.7 \\
\%\end{array}$ & $\begin{array}{c}66 \\
31.4 \\
\%\end{array}$ & $\begin{array}{c}42 \\
20.0 \\
\%\end{array}$ & 4.23 & 1.579 \\
\hline
\end{tabular}


Vol.8, No.5, pp. 56-79, August 2020

Published by ECRTD-UK

Print ISSN: 2053-4019(Print), Online ISSN: 2053-4027(Online)

\begin{tabular}{|c|c|c|c|c|c|c|c|c|c|}
\hline $\begin{array}{l}\text { The company has } \\
\text { tangible fixed assets } \\
\text { suitable as collateral to } \\
\text { guarantee bank loan to } \\
\text { enhance business } \\
\text { operations and } \\
\text { profitability. }\end{array}$ & $\begin{array}{c}14 \\
6.7 \%\end{array}$ & $\begin{array}{c}6 \\
2.9 \%\end{array}$ & $\begin{array}{c}6 \\
2.9 \%\end{array}$ & $\begin{array}{c}18 \\
8.6 \%\end{array}$ & $\begin{array}{c}54 \\
25.7 \\
\%\end{array}$ & $\begin{array}{c}64 \\
30.5 \\
\%\end{array}$ & $\begin{array}{c}48 \\
22.9 \\
\%\end{array}$ & 4.27 & 1.638 \\
\hline $\begin{array}{l}\text { My company's current } \\
\text { asset always places it } \\
\text { at advantage for } \\
\text { business. }\end{array}$ & $\begin{array}{c}16 \\
7.6 \%\end{array}$ & $\begin{array}{c}2 \\
1.0 \%\end{array}$ & $\begin{array}{c}12 \\
5.7 \%\end{array}$ & $\begin{array}{c}22 \\
10.5 \%\end{array}$ & $\begin{array}{c}46 \\
21.9 \\
\%\end{array}$ & $\begin{array}{c}76 \\
36.2 \\
\%\end{array}$ & $\begin{array}{c}36 \\
17.1 \\
\%\end{array}$ & 4.15 & 1.636 \\
\hline
\end{tabular}

Key: UD (Undecided), SD (Strongly Disagree), D (Disagree), PD (Partially Disagree), PA (Partially Agree), A (Agree) and SA (Strongly Agree).

Table 3 question (1) on profitability indicated that 12 respondents representing $5.7 \%$ were uncertain that they are satisfied with the profit level of their business, 34 respondents representing $16.2 \%$ strongly disagreed, 20 respondents representing $9.5 \%$ disagreed, 30 respondents representing $14.3 \%$ partially disagreed, 44 respondents representing $21.0 \%$ partially agreed, 48 respondents representing $22.9 \%$ agreed and 22 respondents representing $10.5 \%$ strongly agreed.

Question (2) on profitability revealed that 12 respondents representing 5.7\% were unsure that their company's profitability ratio such as return on investment and return on equity showed that their firm made sustainable profit, 2 respondents representing 1.0\% strongly disagreed, 20 respondents representing $9.5 \%$ disagreed, 22 respondents representing $10.5 \%$ partially disagreed, 46 respondents representing $21.9 \%$ partially agreed, 76 respondents representing $36.2 \%$ agreed and 32 respondents representing $15.2 \%$ strongly agreed.

Question (3) on profitability showed that 20 respondents representing 9.5\% were unsure that earnings per share of their firm increased, 2 respondents representing 1.0\% strongly disagreed, 24 respondents representing $11.4 \%$ disagreed, 28 respondents representing $13.3 \%$ partially disagreed, 46 respondents representing $21.9 \%$ partially agreed, 60 respondents representing $21.6 \%$ agreed and 30 respondents representing $14.3 \%$ strongly agreed.

Question (4) on profitability indicated that 20 respondents representing $9.5 \%$ were uncertain that their firms' overall financial performance was acknowledged by its bank(s), 6 respondents representing $2.9 \%$ strongly disagreed, 16 respondents representing $7.6 \%$ disagreed, 36 respondents representing $17.1 \%$ partially disagreed, 44 respondents representing $21.0 \%$ partially agreed, 48 respondents representing $22.9 \%$ agreed and 40 respondents representing $19.0 \%$ strongly agreed. 
Question (5) on sales growth revealed that 14 respondents representing 6.7\% were uncertain that since take off of their business, sales level has grown significantly, 8 respondents representing $3.8 \%$ disagreed, 22 respondents representing $10.5 \%$ partially disagreed, 50 respondents representing $23.8 \%$ partially agreed, 70 respondents representing $33.3 \%$ agreed and 46 respondents representing $21.9 \%$ strongly agreed.

Question (6) on sales growth showed that 14 respondents representing 6.7\% were uncertain that there has been an increase in the number of people who were willing to sell and patronize their company's goods/services, 2 respondents representing 1.0\% strongly disagreed, 6 respondents representing $2.9 \%$ disagreed, 18 respondents representing $8.6 \%$ partially disagreed, 32 respondents representing $15.2 \%$ partially agreed, 92 respondents representing $43.8 \%$ agreed and 46 respondents representing $21.9 \%$ strongly agreed.

Question (7) on sales growth revealed that 16 respondents representing $7.6 \%$ were unsure that their company's sales strategy was responsible for its increased revenue through enhanced sales, 8 respondents representing 3.8\% strongly disagreed, 4 respondents representing $1.9 \%$ disagreed, 18 respondents representing $8.6 \%$ partially disagreed, 40 respondents representing $19.0 \%$ partially agreed, 86 respondents representing $41.0 \%$ agreed and 38 respondents representing $18.1 \%$ strongly agreed.

Question (8) on sales growth indicated that 22 respondents representing $10.5 \%$ were unsure that government policies on patronage of made in Nigeria goods was a boost to sales in their company, 6 respondents representing $2.9 \%$ strongly disagreed, 12 respondents representing $5.7 \%$ disagreed, 16 respondents representing $7.6 \%$ partially disagreed, 34 respondents representing $16.2 \%$ partially agreed, 82 respondents representing $39.0 \%$ agreed and 38 respondents representing $18.1 \%$ strongly agreed.

Question (9) on market share indicated that 18 respondents representing $8.6 \%$ were uncertain that there has been a significant increase in the company's market share, 6 respondents representing $2.9 \%$ strongly disagreed, 8 respondents representing $3.8 \%$ disagreed, 22 respondents representing $10.5 \%$ partially disagreed, 60 respondents representing $28.6 \%$ partially agreed, 60 respondents representing $28.6 \%$ agreed and 36 respondents representing $17.1 \%$ strongly agreed.

Question (10) on market share showed that 14 respondents representing 6.7\% were unsure that their company has expanded to other products and markets, 18 respondents representing $8.6 \%$ strongly disagreed, 22 respondents representing $10.5 \%$ disagreed, 8 respondents representing $3.8 \%$ partially disagreed, 60 respondents representing $28.6 \%$ partially agreed, 58 respondents representing $27.6 \%$ agreed and 30 respondents representing $14.3 \%$ strongly agreed. 
Question (11) on market share indicated that 12 respondents representing 5.7\% were uncertain that their company's product(s)/service(s) had taken over a large chunk of the market in its immediate environment and beyond, 8 respondents representing 3.8\% strongly disagreed, 24 respondents representing $11.4 \%$ disagreed, 28 respondents representing $13.3 \%$ partially disagreed, 40 respondents representing $19.0 \%$ partially agreed, 62 respondents representing $29.5 \%$ agreed and 36 respondents representing $17.1 \%$ strongly agreed.

Question (12) on market share indicated that 14 respondents representing 6.7\% were unsure that their company's products/services enjoyed a wider acceptance compared to when their products/services were introduced, 4 respondents representing $1.9 \%$ strongly disagreed, 10 respondents representing $4.8 \%$ disagreed, 18 respondents representing $8.6 \%$ partially disagreed, 46 respondents representing $21.9 \%$ partially agreed, 72 respondents representing $34.3 \%$ agreed and 46 respondents representing $21.9 \%$ strongly agreed.

Question (13) on net asset growth indicated that 14 respondents representing $6.7 \%$ were unsure that their business had experienced considerable growth in net asset, 2 respondents representing $1.0 \%$ strongly disagreed, 4 respondents representing $1.9 \%$ disagreed, 20 respondents representing $9.5 \%$ partially disagreed, 60 respondents representing $28.6 \%$ partially agreed, 66 respondents representing $31.4 \%$ agreed and 44 respondents representing $21.0 \%$ strongly agreed.

Question (14) on net asset growth showed that 14 respondents representing 6.7\% were uncertain that the net asset of their company and its liability were healthy enough to guarantee success, 14 respondents representing $6.7 \%$ disagreed, 18 respondents representing $8.6 \%$ partially disagreed, 56 respondents representing $26.7 \%$ partially agreed, 66 respondents representing $31.4 \%$ agreed and 42 respondents representing $20.0 \%$ strongly agreed.

Question (15) on net asset growth indicated that 14 respondents representing $6.7 \%$ were unsure that their company had tangible fixed assets suitable as collateral to guarantee bank loan to enhance business operations and profitability, 6 respondents representing $2.9 \%$ strongly disagreed, 6 respondents representing $2.9 \%$ disagreed, 18 respondents representing $8.6 \%$ partially disagreed, 54 respondents representing $25.7 \%$ partially agreed, 64 respondents representing $30.5 \%$ agreed and 48 respondents representing $22.9 \%$ strongly agreed.

Question (16) on net asset growth indicated that 16 respondents representing $7.6 \%$ were unsure that their company's current asset always placed it at advantage for business, 2 respondents representing $1.0 \%$ strongly disagreed, 12 respondents representing $5.7 \%$ disagreed, 22 respondents representing $10.5 \%$ partially disagreed, 46 respondents representing $21.9 \%$ partially agreed, 76 respondents representing $36.2 \%$ agreed and 36 respondents representing $17.1 \%$ strongly agreed.

Combining results in Tables 1, 2 and 3 together, it can be seen that entrepreneurial orientation and entrepreneurial self-efficacy have positive effect on entrepreneurial success. This provides 
answer to the research question and also enables us to achieve the research objective in this study.

Hypothesis (H04): Entrepreneurial capability has no significant effect of entrepreneurial success. The hypothesis is tested using the simple regression analysis. The data for entrepreneurial capability is created by combining all responses for entrepreneurial orientation and self-efficacy while data for entrepreneurial success is created by summing responses of the items for each of the variable. The results of the regression are presented in Tables 4.

Table 4: Relationship between Entrepreneurial Capability and Entrepreneurial Success

\begin{tabular}{|c|c|c|c|c|c|c|}
\hline \multicolumn{7}{|c|}{ Coefficients $^{\mathbf{a}}$} \\
\hline \multirow{2}{*}{\multicolumn{2}{|c|}{ Model }} & \multicolumn{2}{|c|}{$\begin{array}{l}\text { Unstandardized } \\
\text { Coefficients }\end{array}$} & \multirow{2}{*}{$\begin{array}{c}\begin{array}{c}\text { Standardized } \\
\text { Coefficients }\end{array} \\
\text { Beta }\end{array}$} & \multirow[t]{2}{*}{$\mathbf{T}$} & \multirow[t]{2}{*}{ Sig. } \\
\hline & & $\mathbf{B}$ & $\begin{array}{c}\text { Std. } \\
\text { Error }\end{array}$ & & & \\
\hline \multirow[t]{2}{*}{1} & (Constant) & -5.065 & 9.106 & & -.556 & .579 \\
\hline & $\begin{array}{l}\text { Entrepreneurial } \\
\text { Capability }\end{array}$ & .624 & .080 & .475 & 7.785 & .000 \\
\hline
\end{tabular}
$F(2,207)=60.605, p=0.000, R^{2}=.226, R^{2}$ Adjusted $=.222$

Table 4 shows regression analysis of the relationship between entrepreneurial capability and entrepreneurial success. The result of the regression analysis indicated that entrepreneurial capability has a strong positive relationship with entrepreneurial success $(R=.475)$. The coefficient of determination $\mathrm{R}^{2}$ is .226 , suggests that $22.6 \%$ of the variation (or changes) in entrepreneurial success of selected SMEs in Lagos State is explained by entrepreneurial capability. The value of $F(1,208)=60.605, p<.05$, demonstrates that entrepreneurial capability statistically and significantly predicts the entrepreneurial success and that entrepreneurial capability significantly influence entrepreneurial success in Lagos State. The coefficient of entrepreneurial capability is statistically significant $(\mathrm{t}=7.785, p<.05)$. The regression model explaining the results in Table 4 is therefore given by:

ENT_SU $=-5.065+0.624 E N T \_C A P$

Where: ENT_SU = Entrepreneurial Success

ENT_CAP = Entrepreneurial Capability

The regression model shows that entrepreneurial capability positively affects the entrepreneurial success. The model shows that a unit change in entrepreneurial capability led to 0.624 units of positive change in entrepreneurial success of selected SMEs in Lagos State. The p-value of entrepreneurial capability is .000 which is less than the 0.05 level of significance adopted for this study. This suggests that entrepreneurial capability is significant in influencing the 
entrepreneurial success of selected SME's in Lagos State positively. Based on these findings, the null hypothesis four $\left(\mathrm{H}_{04}\right)$ which states that entrepreneurial capability has no significant effect on entrepreneurial success is hereby rejected.

\section{DISCUSSION}

The findings of the hypothesis show that entrepreneurial capability has a significant effect on entrepreneurial success. It indicates that entrepreneurial orientation and entrepreneur's taskspecific self-confidence determines business success. The finding is supported by Oyeku (2014) who upheld that behind the successful performance of an enterprise is the entrepreneur and the capabilities required to carry on his business successfully in a constantly changing or dynamic business environment remain his competency, orientation and self-efficacy. The findings are in line with Yang Yang (2014) who studied the relationship between entrepreneurial capability and corporate performance with significant positive correlation between entrepreneurial capability and corporate performance.

The findings are also in line with previous empirical studies on significant positive effects of entrepreneurial capability on entrepreneurial success or firms' performance (Woldesenbet, Ran and Jones, 2012; Man, Lau and Chan, 2002; Man, Lau and Chan, 2000; Zhang, Tansuhaj and McCulloughe, 2009; Zahra, Abdelgawad and Tsang, 2011; and Abdelgawad, et al., 2013). JemiAlade (2013), acknowledged that the major challenge facing SMEs in Nigeria is increasing rate of business failure which he attributed to low level of entrepreneurial orientation.

Covin and Miles (1999) in a study on conceptual model of entrepreneurship as firm behaviour found that firms with high levels of entrepreneurial orientation tend to constantly scan and monitor their operating environment in other to find new opportunities and strengthen their competitive positions and improve their performance. Liu (2004) in a comparative research on the competitiveness of Shaoxing textile enterprises cluster found that in a dynamic and complex industry, entrepreneurial orientation is required for successful business performance. Madhoushi, Sadti, Delavari, Mehdivand and Mihandost (2001) studied the relationship between entrepreneurial orientation, knowledge management and innovation performance using random sampling technique to select 365 SMEs who are at least ten years old from the SMEs operating in the industrial zone of Mazandaran, Iran. The study found a positive relationship between entrepreneurial orientation and innovation performance as well as positive relationship between entrepreneurial orientation and knowledge management.

In the area of self-efficacy, Markman, Balkin and Baron (2002) described self-efficacy as a key determinant of new venture growth and personal success. Shane, Locke and Collins (2003) examined entrepreneurial motivation and found that an entrepreneur who is high in self-efficacy is likely to "exert more effort for a greater length of time, persist through setbacks, and develop better plans and strategies for the task (emphasis added). Hmieleski and Corbett (2008) have 
established a positive relationship between the self-efficacy of entrepreneurs and the growth of their firms. Forbes (2005) and Anna, Chandler, Jansen and Mero (2000) have found a positive relationship between entrepreneurial self-efficacy and subjective measures of new venture performance. Torres and Watson (2013) in their study on an examination of the relationship between manager Self-efficacy and entrepreneurial Intentions and performance of Mexican small businesses observed that high performance requires higher belief levels of the owner or manager about his or her capacity to perform and high involvement tasks and roles as well as noting that only one of the three self-efficacy factors.

The study is in line with Personality Trait Theory. Personality theory emphasized personal characteristics that define entrepreneurship; such characteristics include: need for achievement, locus of control risk taking, innovativeness, and tolerance for ambiguity. This theory believes that these characteristics are unique to entrepreneurs and a combination of these attributes stand to distinguish entrepreneurs from non-entrepreneurs.

The findings of these studies suggest that entrepreneurs high in self-efficacy are likely to set challenging growth expectations for their firms and persist in their leadership efforts toward the accomplishment of those goals. Based on the findings and its supporting literature the study therefore rejects the null hypothesis $\left(\mathrm{H}_{04}\right)$ which states that entrepreneurial capability has no significant effect on entrepreneurial success with p-value of 0.000 which is greater the adopted level of significance 0.05 .

\section{Limitations and Suggestions for Future Research}

This study is not without limitations. These limitations however, present opportunities for future research. The research design approach is cross sectional survey making it impossible to collect data over a long period of time for a more robust interaction with the respondents resulting in more reliable results that could be generalized. Also, this study can be carried out in different sectors, segments and category of entrepreneurs to be industry specific. This would provide opportunity to evaluate entrepreneurial success factors industry by industry amongst the small and medium enterprises. Another limitation in this study could be in the area of sample size. Only 381 samples out of 9,450 registered entrepreneurs with relevant BMOs in Lagos state were used for this study. This could actually limit the extent to which we can generalize the results of the study.

\section{CONCLUSION}

The findings of this study are consistent with literature. The study shows that entrepreneurial capability has a significant and positive relationship with entrepreneurial success in Lagos State, Nigeria. This implies that the combined effect of entrepreneurial orientation and entrepreneurial self efficacy is statistically significant in explaining changes in entrepreneurial success. The findings of the study suggest that entrepreneurs who display high level of entrepreneurial 
capability are likely to succeed in operating their enterprises. The findings of the study could be of great benefits to all existing entrepreneurs, aspiring entrepreneurs, government, researchers and the general public by having a deeper understanding of critical factors that affects entrepreneurial success. This would assist in increasing industry frontier as well as improve the rate of business survival especially during and after covid-19 pandemic.

\section{REFERENCES}

Abdelgawad, S.G., et al. (2013). Strategic Leadership and Entrepreneurial Capability for Game Change. Journal of Leadership \& Organizational Studies, 20(4), 394-407.

Anna, A.L, Chandler, G.N, Jansen, E. \& Mero, N.P. (2000), Women business owners in traditional and nontraditional industries. Journal of Business Venturing, 15(3), 279-303.

Ayala, J., \& Manzano, G. (2014). The resilience of the entrepreneur. Influence on the success of the business. A longitudinal analysis. Journal of Economic Psychology, 42, 126-135. doi:10.1016/j.joep.2014.02.004

Burger, L., O’Neill, C. \& Mahadea, D. (2005). The impact of previous knowledge on the entrepreneurial attitudes of grade 12 Learners. South African Journal of Education, 25(2), 8994

Butler, J.E., Keh, H.T. \& Chamormarn, W. (2000), Information acquisition, entrepreneurial performance and the evolution of modern Thai retailing. Journal Asian Business, 30, 4153.

Buttner, E.H. (2001), Examining female entrepreneurs' management style: An application of relational frame. Journal of Business Ethics, 29, 253-269.

Chen, G., Gully, S. M. \& Eden, D. (2001). Validation of a new general self-efficacy scale. Organizational Research Methods, 4(1), 62-68.

Clarysse, B., V. Tartari and A. Salter (2011). The impact of entrepreneurial capacity, experience and organizational support on academic entrepreneurship. Research Policy, 40(8), 10841093.

Covin, J.G. \& Slevin, D.P. (1989), Strategic management of small firms in hostile and benign Environments. Strategic Management Journal, 10, 75-87.

Dillman, D. (2000). Constructing the questionnaire: Mail and internet surveys. New York: John Wiley \& Sons.

Dyer, J.H., Greggersen, H.B. \& Christensen, C. (2008). Entrepreneur Behaviours, OpportunityRecognition and the Origins of Innovative Ventures. Strategic Entrepreneurship Journal 2, 317-338.

Forbes, D.P. (2005). The effects of strategic decision making on entrepreneurial Self efficacy. Entrepreneurship Theory and Practice,29(5), 599-626.

He, Xiao-Gang and Gu-Yan Lin (2009)."Venturing Business Diversification: An Empirical Examination Based on Entrepreneurial Competence”, Science \& Technology Progress and Policy, 26(10), 75-80. 
Hmieleski, K.M., \& Baron, R.A. (2008). When does entrepreneurial self-efficacy enhance versus reduce firm performance. Strategic Entrepreneurship Journal, 2: 57-72.

Islam, M.A., Khan, M.A., Obaidullah, A.Z.M. \& Alam, M.S. (2011), Effect of entrepreneur and firm characteristics on the business success of small and medium enterprises (SMEs) in Bangladesh. International Journal of Business and Management, 6 (3), 289-299.

Jemi-Alade. (2013). Acceptance Speech by Jemi-Alade during the Swearing in Ceremony as the Chairman Elect of the National Association of Small and Medium Enterprises (NASME), Lagos State Branch at Akinrele Auditorium, Federal Institute of Industrial Research Oshodi, Lagos on 21st November, 2013 (Unpublished).Lagos: Federal Institute of Industrial Research Oshodi,

Jiao, H., D. Ogilvie and Y. Cui (2010). An empirical study of mechanisms to enhance entrepreneurs' capabilities through entrepreneurial learning in an emerging market. Journal of Chinese Entrepreneurship, 2(2), 196-217.

Kotey, B., Sharma, B. \& Gao, K. (2013), The configuration of entrepreneurial strategy in Chinese small textile manufacturing firms. Journal of New Business Ideas \& Trends, 11(1), 1-19.

Liu, X. (2004), The comparative research on the competitiveness of Shaoxing textile enterprises Cluster. Journal of Wanli College Zhejiang 6, 33-50.

Madhoushi, M., Sadti, A., Delavari, H., Mehdivand, M. \& Mihandost, R. (2001), Entrepreneurial Orientation and Innovation Performance: The Mediating Role of Knowledge Management. Asian Journal of Business Management, 3(4), 310-316.

Man, T.W.Y., T. Lau and K.F. Chan (2002). The competitiveness of small and medium enterprises: A conceptualization with focus on entrepreneurial competencies. Journal of Business Venturing, 17(2), 123 - 142.

Man, T.W.Y. and T. Lau (2002). Entrepreneurial competencies of SME owner/managers in the Hong Kong services sector: A qualitative analysis. Journal of Enterprising Culture, 8(3), 235-254.

Markman, G.D., Balkin., D.B. \& Baron, R.A. (2002), Inventors and new venture formation: the effects of general self-efficacy and regretful thinking. Entrepreneurship Theory and Practice, 27 (2), 149-66.

Miller, D. and Friesen, P.H. (1982). Innovation in conservative and entrepreneurial firms: two models of strategic momentum. Strategic Management Journal, 3, 1-25.

Murphy, G.B. \& Callaway, S.K. (2004). Doing well and happy about it? Explaining variance in entrepreneurs' stated satisfaction with performance. New England Journal of Entrepreneurship, 7, 15-26.

Obschonka, M., et al. (2011). Nascent entrepreneurship and the developing individual: Early entrepreneurial competence in adolescence and venture creation success during the career. Journal of Vocational Behavior, 79(1), 121-133.

Ohyama, A., S. Braguinsky and K.M. Murphy (2004) Entrepreneurial ability and market selection in an infant industry: evidence from the Japanese cotton spinning industry. Review of Economic Dynamics, 7(2), 354-381. 
Owoseni, O.O. \& Akanbi, P.A. (2011). An investigation of personality on entrepreneurial success, Journal of Emerging Trends in Economics and Management Sciences (JETEMS), 2 (2), 95-103.

Oyeku O.M, Oduyoye O.O, Elemo G.N, Akindoju A.P, Karimu F.A, and Unuigbe K.O (2015). Chemistry Entrepreneurship for Small and Medium Enterprises Development: A Panacea for Job and Wealth Creation. Industrial Engineering Letters, 5(4): 1-11.

Oyeku, Oyedele M; Oduyoye, Oluseyi O; Ashikia Olalekan; Kabuoh Margaret and Elemo Gloria N (2014). On Entrepreneurial Success of Small and Medium Enterprises (SMEs): A Conceptual and Theoretical Framework. Journal of Economics and Sustainable Development, 5 (17): 14-23.

Oyeku, O.M; Oduyoye O.O; Kabuoh M; Elemo, G.N; Karimu, A.F; and Akindoju, A.F (2014). On Entrepreneurial Self Efficacy and Entrepreneurial Success: A Conceptual and Theoretical Framework. European Journal of Business and Management, 6 (26): 95-102.

Oyeku, O.M; Oduyoye O.O; Elemo G.N; Akindoju A.F and Karimu, F.A (2014). Entrepreneurial Capability and Entrepreneurial Success: A Review of Conceptual and Theoretical Framework. Research on Humanities and Social Sciences, 4 (17): 136-143.

Perez, E.H., \& Canino, R.M.B. (2009), The Importance of the entrepreneur's perception of Success. Review of International Comparative Management, 10 (5), 990-1003.

Phillips N and Tracey P. (2007). Opportunity recognition, entrepreneurial capabilities and bricolage: connecting institutional theory and entrepreneurship in strategic organization. Strategic Organization, 5 (3), 313-320. DOI: 10.1177/1476127007079956.

Razak, M. R. A., Said, A. A., Ahmad, M. A. H., \& Jumain, R. S. A. (2017). Factors influencing entrepreneurial success of micro-entrepreneur: Partial least square (SEM-PLS). Science International, 29(4), 943.

Sarwoko, E., Surachman. \& Hadiwidjojo, A.D. (2013), Entrepreneurial characteristics and competency as determinant of business performance in SMEs, Journal of Business and Management, 7(3), 31-38.

Setyawati, S.M., Shariff, M.N.M. \& Saud, M.B. (2011). Effects of learning, networking andinnovation adoption on successful entrepreneurs in Central Java, Indonesia. International Journal of Business and Social Science, 2(5).

Shane S. \& Venkataraman, S. (2003). A general theory of entrepreneurship: the individual opportunity Nexus. Massachusetts: Edward Elgar Publishing, Inc.

Staniewski, M. W., Janowski, K., \& Awruk, K. (2016). Entrepreneurial personality dispositions and selected indicators of company functioning. Journal of Business Research, 69(5), 1939-1943. doi:10.1016/j.jbusres.2015.10.084

Torres, J. \& Watson, W. (2013). An Examination of the relationship between manage selfefficacy and entrepreneurial intentions and performance of Mexican small businesses. Contaduriay Administration,58 (3), 65-87.

Wei-Wen, W. (2009). A competency-based model for the success of an entrepreneurial start-up. WSEAS. Transactions on Business and Economics, 6 (6),279-291. 
Wiklund, J. (1999). The sustainability of entrepreneurial orientation -performance relationship. Entrepreneurship Theory and Practice, 24, 37-48.

Windapo, A. (2018). Entrepreneurial factors affecting the sustainable growth and success of a South African construction company. Sustainability 2018, 10(4), 1-11. doi:10.3390/su10041276

Woldesenbet, K., M. Ram and T. Jones (2012). Supplying large firms: The role of entrepreneurial and dynamic capabilities in small businesses. International Small Business Journal, 30(5), 493 - 512.

Yang, Yang (2014), "Entrepreneur Ability, Innovation Competence and EnterprisePerformance", Academi Exploration, 10, 138-142.

Yao, Xiang and Yan-Mei Xu (2013), "Study on the Relationship of Entrepreneur Social Capital, Enterprise Dynamic Capability and Firm Performance", Mathematics in Practice and Theory,

43(9), 66-79.

Zhang, M., P. Tansuhaj and J. McCullough (2009). International Entrepreneurial Capability: Themeasurement and a comparison between born global firms and traditional exporters in China. Journal of International Entrepreneurship, 7, 292-322.

Zahra, S.A., S.G. Abdelgawad and E.W.K. Tsang 2011). Emerging Multinationals Venturing Into Developed Economies: Implications for Learning, Unlearning, and Entrepreneurial Capability. Journal of Management Inquiry, 20(3), 323-330.

Zhang, W., et al. (2010), Entrepreneurial talent and economic development in China. SocioEconomic Planning Sciences, 44(4), 178-192. 